\title{
Archéologie du christianisme éthiopien : quinze ans de collaboration scientifique entre le Centre français d'études éthiopiennes et l'Inrap
}

Marie-Laure Derat, François-Xavier Fauvelle-Aymar, Anne-Marie Jouquand et Bertrand Poissonnier

\section{(2) OpenEdition}

Journals

Édition électronique

URL : https://journals.openedition.org/archeopages/749

DOI : 10.4000/archeopages.749

ISSN : 2269-9872

Éditeur

INRAP - Institut national de recherches archéologiques préventives

Édition imprimée

Date de publication : 1 octobre 2010

Pagination : $37-41$

ISSN : $1622-8545$

\section{Référence électronique}

Marie-Laure Derat, François-Xavier Fauvelle-Aymar, Anne-Marie Jouquand et Bertrand Poissonnier, "Archéologie du christianisme éthiopien : quinze ans de collaboration scientifique entre le Centre français d'études éthiopiennes et l'Inrap ", Archéopages [En ligne], Hors-série 2 | 2010, mis en ligne le 01 octobre 2010, consulté le 23 février 2023. URL : http://journals.openedition.org/archeopages/749 ; DOI : https://doi.org/10.4000/archeopages.749 
Nous projetons l'ouverture du complexe D du secteur 2 afin de caractériser les industries encore mal connues à la charnière des Aurignaciens/ Ahmariens et de l'Épipaléolithique.

BOËDA É., BONILAURI S. 2006 : « The intermediate paleolithic: the first bladelet production 40.00o years ago ", Anthropologie, XLIV/1, p. 75-92. Ploux S., Soriano S., 2003 : «Umm el Tlel, Une séquence du paléolithique supérieur en Syrie centrale. Industries lithiques et chronologie culturelle» Paléorient, vol. 29/2, p. 5-34, CNRS Éditions.

Suzuki H., Kobori I., 1970, « Report of the Reconnaissance Survey on Palaeolithic Sites in Lebanon and Syria », Bulletin, I, Tokyo, University Museum.

\section{Archéologie du christianisme éthiopien : quinze ans de collaboration scientifique entre le Centre français d'études éthiopiennes et l'Inrap}

\author{
Marie-Laure Derat \\ CNRS, Centre francais détudes éthiopiennes, Addis-Abeba (USR 3137) \\ François-Xavier Fauvelle-Aymar \\ CNRS, TRACES-UMR 5608, Toulouse, et chercheur honoraire \\ à la School of Geography, Archaeology and Environmental Studies \\ de l'université du Witwatersrand (Johannesburg, Afrique du Sud) \\ Anne-Marie Jouquand \\ Inrap, UMR 6173 CITERES « Laboratoire archéologie et territoire» \\ Bertrand Poissonnier \\ Inrap
}

D epuis une quinzaine d'années, des recherches archéologiques conduites sous l'égide du Centre français d'études éthiopiennes (CFEE) ont contribué à revisiter le christianisme éthiopien et son contexte géographique et historique. De nombreux archéologues de l'Afan puis de l'Inrap ont participé à ces recherches, qui sont désormais inscrites dans le cadre d'un partenariat scientifique. D'autres collaborations ont également été mises en place au cours des dernières années, notamment au sujet de la préhistoire récente de la Corne de l'Afrique et dans le champ de recherche de l'islam ancien, encore relativement neuf dans cette région du monde ${ }^{2}$. Mais les pages qui suivent ont surtout pour objectif de mettre en relief l'apport de la collaboration de longue haleine pratiquée entre chercheurs de diverses disciplines et institutions sur le terrain spécifique de l'Éthiopie chrétienne médiévale.
Les lacunes documentaires du christianisme éthiopien. Christianisée au IV ${ }^{\mathrm{e}}$ siècle, l'Éthiopie est sans conteste l'une des régions du monde ayant connu la plus longue tradition chrétienne continue, puisque c'est toujours dans cette tradition de christianisme monophysite (historiquement liée à l'Église copte d'Égypte) que vivent la majorité des habitants des hauts plateaux. Et pourtant le christianisme éthiopien souffre de sérieuses lacunes documentaires. Ainsi est-il remarquable que la considérable documentation manuscrite conservée dans les monastères du pays se rapporte, à de très rares exceptions près, à la période postérieure au XIII ${ }^{\mathrm{e}}$ siècle, faisant suite à l'apparition de la dynastie royale des Salomoniens. Quant à l'archéologie des périodes médiévales et modernes, elle présente un tableau mitigé : très peu de sites à vocation liturgique ont été fouillés, principalement pour la raison qu'une tradition vivante continue généralement de les animer et les rend inaccessibles à des investigations poussées (les études de l'architecture et des peintures posent moins de problème à cet égard), et aucun site « civil » n'a encore été identifié sur le terrain pour la période antérieure à la fixation des rois à Gondar (XVII ${ }^{\mathrm{e}}$-XVIII ${ }^{\mathrm{e}}$ siècles). Il est ainsi paradoxal que la période des $\mathrm{XIII}^{\mathrm{e}}-\mathrm{XV}^{\mathrm{e}}$ siècles, période de formidable dynamisme du monachisme éthiopien et d'expansion du royaume chrétien, ne puisse encore faire l'objet d'une approche nourrie d'archéologie. Durant cette période, le camp royal est itinérant. Le roi et sa cour, sans doute plusieurs milliers de personnes, composée de dignitaires civils et religieux, d'hommes de guerres, de serviteurs et d'esclaves, se déplacent presque chaque année au gré des guerres et des nécessités matérielles (nourriture et bois) dans un endroit différent pour la saison des pluies. On possède plusieurs sources qui décrivent ce camp, mais sans doute la description de Francisco Alvares, qui fait partie de l'ambassade portugaise dans l'Abyssinie des années 1520-1526, est-elle l'une des plus vivantes. Ces déplacements sur un vaste territoire s'accompagnent de créations d'églises et de monastères organisés en réseaux, sur lesquels s'appuie le pouvoir royal et qui sont mentionnés dans les sources écrites sans que l'on sache toujours les localiser précisément.

Pour la période antérieure, celle que l'on pourrait appeler le « Haut-Moyen Âge » éthiopien, la situation documentaire est encore plus critique. Les sociétés d'Éthiopie ne sont alors connues que par quelques mentions dans des sources extérieures, chrétiennes d'Égypte ou musulmanes, et seuls de rares témoins de cette période, architecturaux, archéologiques ou de la culture matérielle, ont survécu. C'est en somme pour la période de l'Antiquité tardive ( $\mathrm{IV}^{\mathrm{e}}-\mathrm{VIII}{ }^{\mathrm{e}}$ siècles) que nous sommes le mieux renseignés : sources externes (grecques le plus souvent), séries numismatiques et inscriptions (en grec, en geez ou en sud-arabique) nous informent sur les noms, titres et faits de quelques souverains au moins nominalement chrétiens des $\mathrm{IV}^{\mathrm{e}}-\mathrm{VI}^{\mathrm{e}}$ siècles, tandis que plusieurs églises fouillées sur les territoires éthiopien ou érythréen attestent l'existence d'une multiplicité

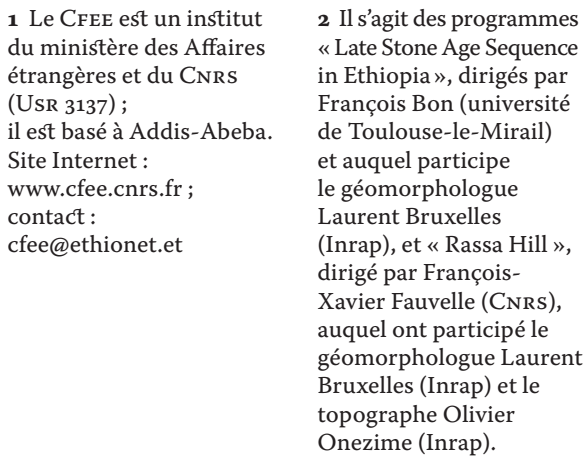


de communautés chrétiennes organisées à partir $\mathrm{du} \mathrm{VI}^{\mathrm{e}}$ siècle.

Ce n'est pas que les lacunes documentaires du christianisme éthiopien soient dues à un défaut d'intérêt pour les traces du passé en général. L'Éthiopie est au contraire une terre bénie de l'archéologie, qu'il s'agisse de l'archéologie des origines de l'homme ou de l'archéologie monumentale, telle qu'elle s'est pratiquée à Axoum et dans d'autres sites tels que Matara ou Adoulis (en Érythrée). C'est en particulier à Axoum, capitale des souverains devenus chrétiens au $\mathrm{IV}^{\mathrm{e}}$ siècle, que se sont rendues plusieurs grandes missions expéditionnaires étrangères (allemande, britanniques, italienne) depuis un peu plus d'un siècle, mais surtout qu'ont travaillé, dans une relative continuité, des chercheurs français depuis les années 1950 (ces travaux sont surtout publiés dans la revue Annales d'Éthiopie, accessible en ligne sur le portail « Persée »). Mais sachons reconnaître que, en dépit de cet intérêt pour les restes archéologiques relativement prestigieux de certaines périodes bien spécifiques (autour des stèles d'Axoum par exemple), l'Éthiopie reste un pays largement sous-documenté sur le plan archéologique. Les raisons en sont multiples : très grandes difficultés logistiques et de transport local, absence d'une politique archéologique et patrimoniale à l'échelle nationale, défaut de formation d'un personnel spécialisé, pratique courante du pillage, barrières traditionnelles s'opposant à l'accès des chercheurs à certains lieux ou sanctuaires.

Signe qu'une prise de conscience est en train de s'opérer dans ce domaine, une « école d'été » (summer school) sur le thème de l'archéologie préventive a reçu en 2008 un écho extrêmement favorable en Éthiopie. Organisé à Axoum, dans la toute jeune université de la ville, qui ouvrait alors le second département d'archéologie du pays (après Addis-Abeba), cet événement provient d'une initiative du CFEE (dirigé par François-Xavier Fauvelle), l'université d'Axoum (et son président Mebratom Mesfin), l'Authority for Research and Conservation of Cultural Heritage (ARCCH, organisme dépendant du ministère éthiopien de la Culture), représenté par le paléontologue Yonas Beyene, et l'Inrap, représenté par Nathan Schlanger. Cette école prit la forme d'une semaine intensive de cours, conférences, travaux de terrain et de laboratoire pour une cinquantaine d'étudiants de l'université, sous la houlette d'archéologues (Bertrand Poissonnier, Inrap ; Tekle Hagos, université d'Addis-Abeba ; Hiluf Berhe, université d'Axoum), d'un topographe (Régis Bernard, Inrap), d'historiennes (Claire Bosc-Tiessé et Marie-Laure Derat, toutes deux CNRs et CFEE) et d'une muséographe (Aurèle Letricot, IsFCT). Dans le contexte déjà signalé, un tel événement ne peut être qu'une pierre d'attente pour l'archéologie éthiopienne, mais il contribue déjà à transmettre une conscience archéologique autant que des savoir-faire techniques. Il participe aussi de la nécessaire promotion d'une politique de prospections et de fouilles, non pas orientée par la perspective d'une rente symbolique immédiate (les « origines » de l'homme, de la civilisation monumentale ou encore du christianisme), mais énoncée en termes de production d'une documentation archéologique.

\section{Le camp royal introuvable, l'église retrouvée.}

Cette perspective documentaire avait été ouverte une dizaine d'années auparavant. De 1998 à 2000, une équipe d'archéologues et d'historiens avait en effet entrepris des fouilles dans la région du Manz, sur un ensemble de sites connus par la tradition pour avoir été un camp royal chrétien du Moyen Âge. Des vestiges d'autres périodes étaient également présents dans les environs immédiats, en particulier une église et une nécropole, ainsi que plusieurs tumulus et sites à stèles de période inconnue. Trois campagnes de fouilles ont été conduites. Les travaux étaient dirigée par Bertrand Hirsch (professeur d'histoire de l'Afrique à Paris I), alors directeur du CFEE, et Bertrand Poissonnier, alors mis à disposition par l'Inrap (à l'époque Afan) auprès de l'ARCCH et basé au CFEE. Les recherches étaient conduites dans le cadre d'un programme ACI du ministère de la Recherche et sur des financements propres du CFEE, avec l'accord et le soutien actif des autorités éthiopiennes de la Culture. Les campagnes de fouilles et les enquêtes historiques ont été menées dans les environs du village de Meshala Maryam (littéralement, le " suaire de Marie »), district du Manz et région du Choa, à environ $320 \mathrm{~km}$ au nord d'Addis-Abeba. Ce site était connu par des textes du $\mathrm{Xv}^{\mathrm{e}}$ siècle comme étant l'une des églises fondées par le roi Ba'eda Maryam au cours de son règne, entre 1468 et 1478. Quoique l'église actuelle de Meshala Maryam fût tout juste reconstruite à l'aide de matériaux modernes, des prospections menées en 1997 avaient confirmé le potentiel du site en identifiant des structures anciennes visibles au sol et des pierres errantes appartenant à un contexte $\mathrm{du} \mathrm{Xv}^{\mathrm{e}}$ siècle. Ces vestiges étaient en outre désignés par les habitants comme les restes du camp du fameux roi.

Les fouilles engagées à partir de 1998 sur le site supposé du camp royal de Meshala Maryam se sont très vite élargies à d'autres sites dont l'importance s'est révélée au cours des campagnes. Ainsi, dès 1999, des tumulus ont été fouillés, dont le mobilier (céramiques, perles, armes) renvoyait au premier millénaire de notre ère. Cette culture à tumulus, baptisée «culture Shay » par Bertrand Poissonnier en raison de sa distribution autour de la rivière du même nom, documentait pour la première fois un horizon chronologique jusqu'alors inconnu pour cette région, celui immédiatement antérieur à la christianisation (Fauvelle-Aymar et al., 2007-2008) (cf. encadré p. 41). Il illustrait aussi la continuité de l'occupation élitaire des lieux, depuis les élites « païennes » du Haut-Moyen Âge, capables d'emporter dans la tombe une abondance de biens de prestige, jusqu'au souverain et à l'église $\mathrm{du} \mathrm{XV}^{\mathrm{e}}$ siècle.

En 200o, un nouveau chantier fut ouvert sur une petite éminence rocheuse ( $a m b a$ ) surplombant
3 Les résultats complets de la fouille du camp royal et de l'église de Gabriel ont fait l'objet d'une monographie à paraitre sous peu : DERAT M.-L., JOUQUAND A.-M. (DIR.), Gabriel, une église médiévale d'Éthiopie. Interprétations historiques et archéologiques de sites chrétiens autour de Meshala Mãryām (XV $V^{e}-X V I I^{e}$ siècles). 
la plaine entourant Meshala Maryam, à la suite d'indications orales permettant de croire à l'existence en cet endroit d'une église dédiée à l'archange Gabriel. Au cours de deux campagnes de fouilles furent en effet dégagées les structures d'une ancienne église détruite au XVI ${ }^{\mathrm{e}}$ siècle par un incendie ainsi que de nombreuses sépultures superposées aux ruines. Un mobilier relativement riche (fragments de céladon chinois, perles de verre, tiges en métal, etc.) fut découvert parmi les restes de l'église, ce qui, par comparaison, faisait cruellement défaut sur le site même du camp royal $\mathrm{du} \mathrm{Xv}$ siècle, dont les restes matériels, il faut bien l'avouer, n'étaient pas à la hauteur des attentes (Hirsch, Poissonnier, 200o) ${ }^{\mathbf{3}}$.

L'église Gabriel, premier référentiel d'Éthiopie. La découverte inattendue de l'église Gabriel et d'une nécropole postérieure allait fournir un référentiel architectural et funéraire d'importance pour l'archéologie du christianisme éthiopien. La fouille réalisée au sommet de l'amba s'est déroulée sur deux campagnes aux printemps 2000 et $2001^{4}$. Toutes les sépultures identifiées au cours de la fouille n'ont pas pu être examinées. Cependant, l'échantillon disponible (une cinquantaine de tombes documentées sur les 90 repérées) apparaît désormais suffisant pour dresser un premier état des lieux sur la question funéraire dans le christianisme médiéval éthiopien, sachant que l'on ne dispose pas d'autre corpus pour cette période. Bernard Farago-Szekeres a entièrement pris en charge le travail d'étude des sépultures, effectuant un catalogue complet des tombes, point de départ d'une analyse sur les différentes périodes d'inhumation des corps. Comme dans le cas des études sur la céramique ou le petit mobilier, l'étude anthropologique a pu être finalisée au cours des dernières années grâce à un projet d'activité scientifique accordé par l'Inrap en soutien à ce programme.

Premier monument religieux fouillé en Éthiopie pour cette période, l'église Gabriel est, par son existence, le témoin d'un processus encore largement invisible dans la documentation à notre disposition, celui de la pénétration et de la consolidation du christianisme au fur et à mesure de l'expansion du royaume d'Éthiopie. Le mobilier dégagé lors des fouilles témoigne des échanges

\footnotetext{
4. Sur le terrain, l'équipe de fouille était constituée d'Anne-Marie Jouquand, de Bernard Farago-Szekeres anthropologue, et de Deresse Ayenachew, secondés par des ouvriers recrutés dans le voisinage. L'ensemble des plans a été réalisé par Régis Bernard. 5 Notamment deux articles sont parus dans la presse scientifique de vulgarisation : juillet 2009, « À Lalibela, il n’y avait pas que des anges...», Sciences et Avenir. octobre 2009, «Lalibela site éthiopien taillé dans le roc », Pour la Science,
}

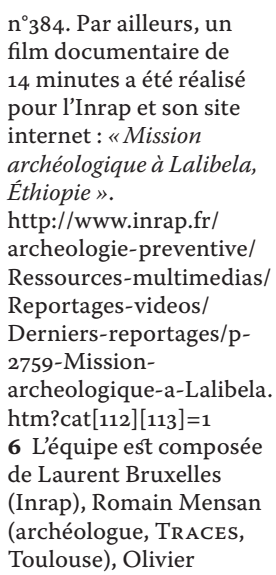

$n^{\circ} 384$. Par ailleurs, un film documentaire de 14 minutes a été réalisé pour l'Inrap et son site internet: "Mission archéologique à Lalibela Éthiopie».

http://www.inrap.fr/ archeologie-preventive/ Ressources-multimedias/ Reportages-videos/ Derniers-reportages/p2759-Mission-

archeologique-a-Lalibela. htm?cat $[112][113]=1$ 6 L'équipe est composée de Laurent Bruxelles (Inrap), Romain Mensan (archéologue, Traces, Toulouse), Olivier

de l'Éthiopie chrétienne avec le reste du monde : les hauts plateaux étaient en quelque sorte la dernière étape d'itinéraires commerciaux mettant en contact aussi bien l'Europe que la Chine avec la Corne de l'Afrique via la mer Rouge. Par sa destruction, elle aussi documentée grâce à la fouille, elle livre un témoignage de la conflagration guerrière qui oppose chrétiens et musulmans au XVI ${ }^{\mathrm{e}}$ siècle. Enfin, par la réoccupation de ses ruines, elle témoigne de la vivacité du christianisme éthiopien et de la mémoire des lieux par-delà les accidents de l'histoire (cf. encadrés p. 43).

Lalibela : au cour de la chrétienté éthiopienne. Depuis 2009, un programme français s'intéresse également à Lalibela, ensemble de plusieurs églises détourées dans la roche et intérieurement excavées, dont la construction est généralement attribuée à un très bref épisode du XIII ${ }^{\mathrm{e}}$ siècle. Conçu dès l'abord en coopération avec l'Inrap, ce programme initié par François-Xavier Fauvelle est aujourd'hui dirigé par Claire Bosc-Tiessé et Marie-Laure Derat. Ce projet ne cache pas son ambition de revisiter le phasage du site et son attribution chronologique, ainsi que d'entreprendre les premières fouilles jamais réalisées sur cet ensemble d'églises rupestres classé sur la liste du Patrimoine mondial de l'Unesco depuis 1978. Deux campagnes ont eu lieu en 2009 et 2010, dont les résultats, déjà remarquables, ont fait l'objet d'une couverture médiatique importante ${ }^{\mathbf{5}}$ et d'un article dans la revue Antiquity (cf. encadré p. 45). Fruit d'une coopération exemplaire entre institutions françaises, éthiopiennes et américaines, cette mission a bénéficié de la mise à disposition d'agents de l'Inrap ainsi que de financements de la commission des Fouilles, du CFEE, de l'AnR Cornafrique et du mécénat privé (compagnie Ethiopian Airlines) ${ }^{\mathbf{6}}$. En 2010, les objectifs de cette mission étaient d'engager des fouilles sur un site voisin des églises de Lalibela (Qedemt) abritant des sépultures anciennes et identifié l'année précédente par l'équipe pour son potentiel archéologique ; d'effectuer des sondages dans les déblais résultant de l'excavation des églises afin d'identifier des niveaux d'occupation, des phases de creusement et des sondages par " géoradar »; d'achever la cartographie du site pour une publication dans l'année et de poursuivre l'observation du phasage de l'excavation du site. Les fouilles menées sur le site de Qedemt ont révélé un cimetière qui a fonctionné sur le long terme. Plusieurs sépultures ont fait l'objet de réemplois : l'une des fosses fouillées a livré, par exemple, les restes de sept corps déposés à des périodes différentes. En l'absence de stratigraphie empêchant de dater ces sépultures, ce sont les datations au carbone 14 des restes osseux qui permettront d'obtenir une séquence chronologique. Les premières observations permettent d'ores et déjà de faire des rapprochements avec le site des églises sur la base de la typologie des tombes, l'intérêt étant d'étudier les peuplements et les pratiques funéraires dans la région et sur le site même des églises de Lalibela, lieu de
Inrap), Matthew Sisk

(archéologue topographe, Yves Gleize (archéo-

anthropologue, Inrap)

François-Xavier Fauvelle

(historien, CNRS),

(liturgiste, CFEE)

Dominique Rousset

(géophysicien, université

de Pau), et de plusieurs

étudiants éthiopiens. 


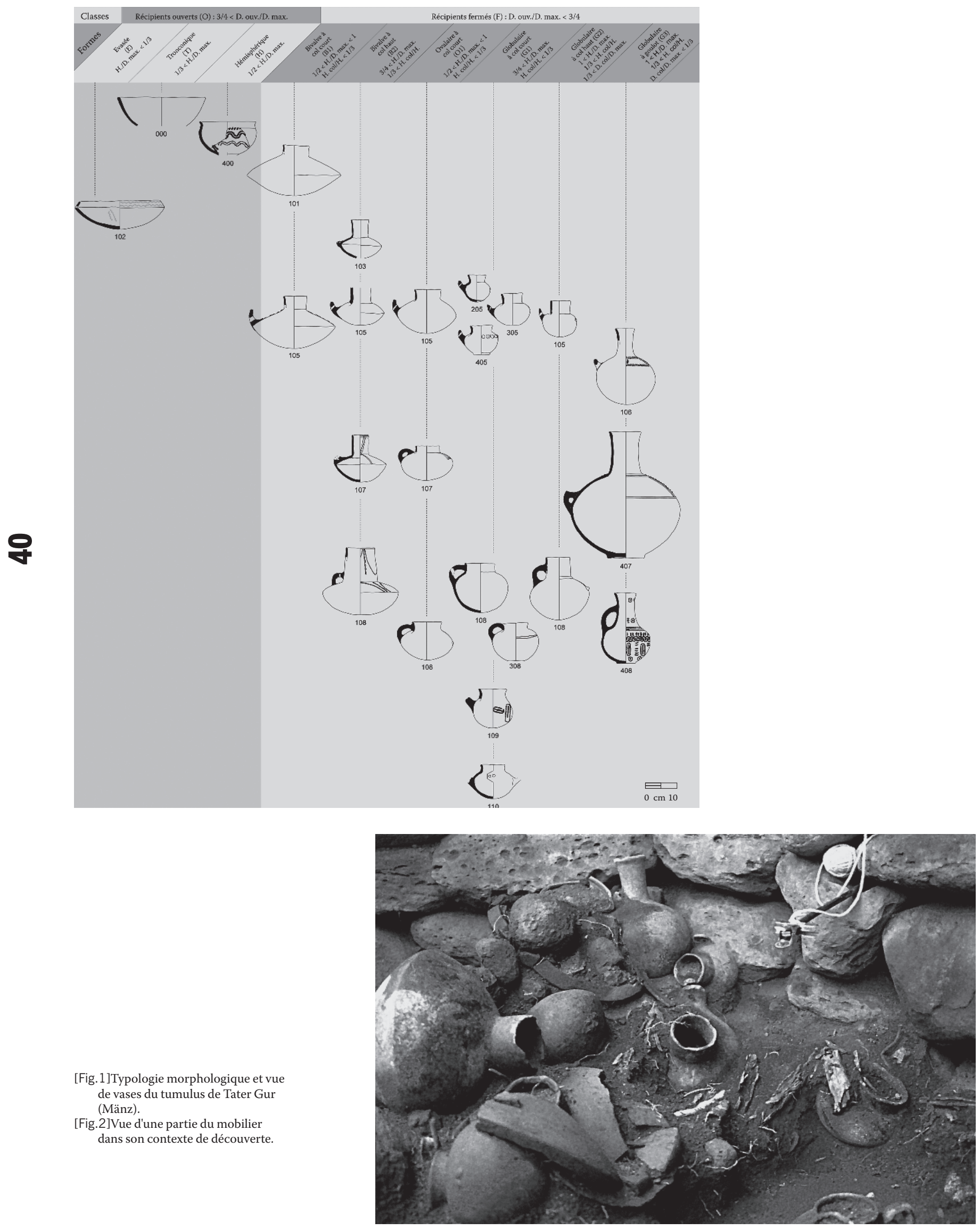


pèlerinage et nécropole sur une longue durée. Les sondages entrepris dans les tas de déblais à Lalibela ont confirmé les observations réalisées l'année précédente sur les étapes de creusement en mettant en évidence différents niveaux de sol qui témoignent des moments de creusement et d'occupation du site. Le travail archéologique est, dans ce cas, mené en parallèle aux recherches historiques dans les archives conservées sur le site à partir du XIII ${ }^{\mathrm{e}}$ siècle. Il apparaît aujourd'hui que Lalibela ne résulte pas d'un programme mené d'un seul tenant, mais d'interventions répétées au cours de plusieurs siècles et qui n'ont pas cessé de modifier le site.

Les recherches archéologiques en Éthiopie, pour les périodes historiques, doivent beaucoup à la collaboration de l'Inrap avec le Centre français des études éthiopiennes. Toutes les campagnes de fouilles menées dans le cadre de cette collaboration ont non seulement ouvert de larges perspectives pour la recherche, mais aussi permis de constituer les premières collections de référence pour l'architecture, le funéraire, la céramique ou pour le lithique. La capacité des archéologues de l'Inrap à s'adapter à tous types de terrain, à fouiller tous types d'époque et de cultures s'avère d'une valeur inestimable. Cette collaboration est essentielle pour combler les lacunes de la recherche sur l'Éthiopie ou sur l'Afrique en général, un domaine dans lequel trop peu d'archéologues sont formés en France, alors qu'il s'agit d'une aire géographique dont le potentiel est immense en terme de découvertes.

CASSEN S., 1995, «Une enquête ethnoarchéologique sur la production céramique de Haro (Éthiopie) ", in Joussaume R. (DIR.), Tiya. l'Éthiopie des mégalithes. Du biface à l'art rupestre dans la Corne de l'Afrique, Chauvigny, Association des Publications Chauvinoises, Mémoire XI, p. 358-373.

Derat M.-L., JouQUAND A.-M.(DIR.), à paraitre : Gabriel, une église médiévale d'Éthiopie. Interprétations historiques et archéologiques de sites chrétiens autour de Meshala Māryām (XV'-XVII ${ }^{\mathrm{e}}$ siècles).

Fauvelle-Aymar F.-X., Ayenachew D., Hirsch B., Bernard R., 2007-2008, «Les monuments mégalithiques du Mänz (nord-Shoa) un inventaire provisoire ", Annales d'Éthiopie, vol. XXIII, p. 329-398.

Hirsch B., Poissonnier B., 200o, « Recherches historiques et archéologiques à Meshalä Māryām (Mänz, Éthiopie) : résultats préliminaires ", Annales d'Éthiopie, 16, p. 59-88.

MunRo-HAY S.C. (DIR.), 1989, Excavations at Aksum. An account of research at the ancient Ethiopian capital directed in 1972-74 by the late Dr Neville Chittick, Londres, British Institute in Eastern Africa, Memoir 10, $359 \mathrm{p}$.

Roux H. DE, 1976, « Aperçu sur la fabrication de la poterie à Yéha (Tigré) », Annales d'Êthiopie, 10, p. 305-320.

\section{La céramique du tumulus de Tater Gur et ses apports pour la connaissance du Haut-Moyen Âge en Éthiopie}

Alain Wittmann

$$
\text { Inrap }
$$

Si le Haut-Moyen Âge éthiopien représente un hiatus dans l'histoire du pays en raison de la rareté des sources écrites, la même méconnaissance pèse sur sa culture matérielle : les sites archéologiques de cette période restent rares et les études de référence sur le mobilier font largement défaut. La fouille récente du tumulus de Tater Gur, dont le fonctionnement a pu être daté entre la fin du $\mathrm{VIII}^{\mathrm{e}}$ et le début du $\mathrm{X}^{\mathrm{e}}$ siècle par une série d'analyses au radiocarbone, s'impose donc comme une étape essentielle dans ce domaine de recherche. Cette tombe privilégiée, constituée d'un couloir d'accès et d'une chambre circulaire architecturés, a livré un dépôt de vases dont l'abondance, l'homogénéité et le remarquable état de conservation justifiaient une étude approfondie. L'ensemble céramique a ainsi offert l'opportunité, non seulement de poser un premier jalon dans l'analyse du répertoire typologique et décoratif régional (culture « Shay »), mais aussi d'aborder des problématiques plus vastes concernant son lien avec les rites funéraires et l'origine géographique de son faciès.

Approches technique et typologique. La mise en parallèle de ce corpus avec des enquêtes ethnographiques conduites sur les sites de production actuels ont permis de restituer, au moins partiellement, la chaîne opératoire qui a présidé au façonnage des vases. En revanche, de nombreux problèmes se posent encore sur l'identification des outils naturels ou anthropiques, voire sur les procédés mis en ouvre pour la réalisation des différentes catégories de décor. Nous avons ensuite élaboré une typologie du mobilier indépendamment de toute considération d'ordre fonctionnel, dans la mesure où la question de l'usage des vases est peu documentée en ethnoarchéologie africaine ; on peut également observer que les céramiques éthiopiennes employées de nos jours n'assurent pas nécessairement une fonction spécifique, mais servent souvent de contenants polyvalents, au point de rendre inopérante la distinction habituelle entre vases à liquides et vases à solides. Le système de classification hiérarchique adopté repose donc uniquement sur des critères morphologiques et métrologiques. Ainsi, les différentes formes se définissent par des rapports de dimensions échafaudés à partir de mesures simples, telles que la hauteur totale, la hauteur du col, le diamètre maximal et le diamètre à l'ouverture [Fig.1]. Bien que cette méthodologie ait été surtout développée pour des productions tournées fortement standardisées, son champ d'application peut s'étendre aux céramiques modelées de Tater Gur, car ces dernières obéissent malgré tout à certaines règles élémentaires de géométrie et se répartissent en séries relativement homogènes. Lavantage majeur d'un tel classement est de fournir une structure ouverte, qui autorisera à tout moment l'intégration de formes et de types nouveaux provenant d'autres sites contemporains de la région du Mänz.

Approches culturelles. Pour l'heure, le rôle, le choix et le traitement de la céramique dans l'appareil funéraire offrent peu de prises à l'interprétation. Les récipients n'avaient-ils qu'une valeur strictement symbolique ou contenaient-ils des denrées alimentaires ? Étaient-ils uniquement destinés au mort et à sa survie dans l'au-delà, ou bien servaient-ils aux vivants pour accomplir leurs 